\title{
Variation of Leptin During Menstrual Cycle and Its Relation to the Hypothalamic-Pituitary-Gonadal (HPG) Axis: A Systematic Review
}

\author{
Ayad Mohammed Salem (iD \\ Department of Physiology, College of \\ Medicine, Imam Abdulrahman Bin Faisal \\ University, Dammam, Saudi Arabia
}

\begin{abstract}
Recently, adipose tissue has been identified as endocrine organ in addition to its action as energy store; it produces a large number of biologically active mediators known as adipocytokines. Significantly, adipocytokines were found to be involved in the physiology of many body functions, including reproduction. The role of body weight, body fat compositions, and nutrition has been largely investigated using animal models and human studies. Malnutrition and/or abnormal body weight may induce disturbances in fertility, puberty, pregnancy, and menstrual cycles. Leptin was the first discovered adipocytokine, and a large body of data over the last 25 years has shown that leptin is not only a molecule that reflects energy stores in the body, but is also an important cytokine involved in many physiological functions, such as inflammatory response, insulin sensitivity, bone metabolism, immunity, and most importantly, reproductive function. Leptin controls the normal physiology of the female reproductive system; it interacts with the hypothalamic-pituitary-gonadal (HPG) axis by a complex mechanism that connects energy homeostasis with reproduction. However, observational studies have demonstrated inconsistent results about leptin variation during normal menstrual cycle, and the mechanisms involved in the interplay between leptin and the hormones of the HPG axis are largely unknown. This review focuses on leptin variation during normal menstrual cycles and its relation to the hypothalamic-pituitary-gonadal axis, and the effect of overweight/obesity on leptin during menstrual cycle is further reviewed.
\end{abstract}

Keywords: leptin, adipocytokines, menstrual cycle, sex hormones, obesity

\section{Introduction}

The physiology of energy homeostasis and the control of appetite have greatly changed since the discovery of leptin, an adipose tissue hormone that was first purified in 1995 . $^{1}$ Recently, a wide range of physiological functions of leptin have been described, and leptin was found to exert a regulatory control upon insulin sensitivity, immune function, reproductive function and a wide range of neuroendocrine axes: ACTH-cortisol, TRH-TSH, prolactin, and GnRH. Leptin signals about the body-fat stores to the hypothalamus and other neuroendocrine centers, and as a feedback response, the neuroendocrine systems modify their function accordingly. ${ }^{2,3}$

Many reviews described the role of leptin in human reproduction comprehensively, showing a key role of leptin in the complex interaction between the nutritional status and the reproductive system. ${ }^{4,5}$ The association between leptin and reproduction was first described by observing that $o b / o b$ gene deficient female mice were obese and sterile. ${ }^{6}$ Humans with congenital leptin deficiency also have obesity and infertilely and
Correspondence: Ayad Mohammed Salem Department of Physiology, College of Medicine, Imam Abdulrahman Bin Faisal University, PO Box 21|4-3|45I,

Dammam, Saudi Arabia

Tel +96655980l 273

Email ayadsalem@iau.edu.sa 
showed failure in puberty development due to hypogonadotropic hypogonadism. ${ }^{7}$ Both obesity and undernutrition were found to affect fertility in humans. ${ }^{8,9}$ Furthermore, leptin play a permissive action for puberty both in girls and boys with obesity, specifically by affecting the hypothalamic-pituitarygonadal axis. ${ }^{10}$

Leptin has a role in the physiology of female reproduction in particular, as it showed gender-related differences at birth, which persist throughout life with a circulating level two to three times higher in females than in males. ${ }^{5}$ Serum leptin in women with hypothalamic amenorrhea was lower than in healthy control women, and administration of leptin restored the menstrual cycles and fertility. ${ }^{11}$ Recently, several studies showed the involvement of leptin in the development of several pregnancy-related diseases such as gestational diabetes. $^{12-14}$ All these findings led to the development of the hypothesis for leptin as an important player in the female reproduction system via its stimulatory effect on the hypothalamic-pituitary-gonadal axis. ${ }^{3,15}$

The role of leptin in the menstrual cycle and female reproduction was first suggested in the late 1990s; several studies noticed a significant variation in serum leptin during the female menstrual cycle, ${ }^{16-20}$ but this variation was absent in postmenopausal women. ${ }^{20}$ Subsequently, many studies showed inconsistent leptin behavior throughout the menstrual cycle, and some reported steady increments in leptin levels from the follicular phase to reach a peak in luteal phase, ${ }^{21-24}$ and others showed a peak during the preovulatory phase $^{25}$ while a stable level of leptin across menstruation was also described. ${ }^{26-28}$ However, this discrepancy highlights a certain role of leptin signaling during the menstrual cycle, but the mechanism of this role is still to be explored.

Currently, there is no review that explores specifically the variation of leptin during the menstrual cycle and its relation to the hypothalamic-pituitary-gonadal axis. In addition, the effect of body weight on leptin during menstrual cycle is not fully elucidated. Thus, the aim of this review is to examine the variation of leptin during the menstrual cycles and its relation to the hypothalamic-pituitary-gonadal axis and to body weight. This review was carried out according to the guidlines provided by the Preferred Reporting Items of Systematic Reviews and Meta-analyses (PRISMA) statement. ${ }^{29}$

\section{Methods}

\section{Information Sources and Search Strategy}

Medline/PubMed and Google Scholar databases were searched for studies of leptin during normal menstrual cycle since 1995 (the time of leptin discovery) until October 2020. The search strategy used keywords related to leptin and menstrual cycle (adipokines[Title] OR adipocytokines[Title]) OR adipose tissue hormones[Title]) OR leptin[Title]) AND menstrual cycle[Title/Abstract]) OR Endometrial Cycle[Title/Abstract]) OR ovarian cycle[Title/ Abstract]) AND "humans"[MeSH Terms]). Further searching through ISI and Scopus and by manual screening the bibliographic references of the selected studies did not yield any additional papers. No language restriction was applied, and the retrieved references were imported into EndNote X5.

\section{Study Selection}

The titles were screened for relevance to the aims and scope of the current review; those studies falling within the scope and were further screened by abstract to determine the eligibility according to inclusion and exclusion criteria. The following inclusion criteria were applied: (a) prospective observational studies that had been run over one or more complete normal menstrual cycles; (b) the study population were adult healthy women with regular menstrual cycles; (c) the primary outcome of the studies was the variation of leptin levels during different phases of the menstrual cycle, with or without investigation of the relationship between leptin and the body weight (BMI); (d) leptin and other hormones assays were conducted in blood or saliva samples during different phases of the menstrual cycle. The current review excluded: (a) studies evaluating irregular menstrual cycles; (b) menstrual cycle in females with associated gynecological diseases or comorbidities, eg, polycystic ovary syndrome (PCOS); (c) studies with less than one complete cycle duration; and (d) randomized clinical studies RCT, review, systematic review, meta-analysis, case report, comments, editorials, letters and animal studies. After the preliminary eligibility screening evaluation, the full articles of the selected studies were obtained (Figure 1).

The following data were extracted from the selected studies:

- General information about the study: author(s), journal name, title, publication year, study design, and location of the study

- Age of the subjects

- Number of participants

- Duration of the study (number of cycles evaluated)

- Frequency of leptin sampling during each cycle

- Major outcomes and conclusion

- Limitations if any 

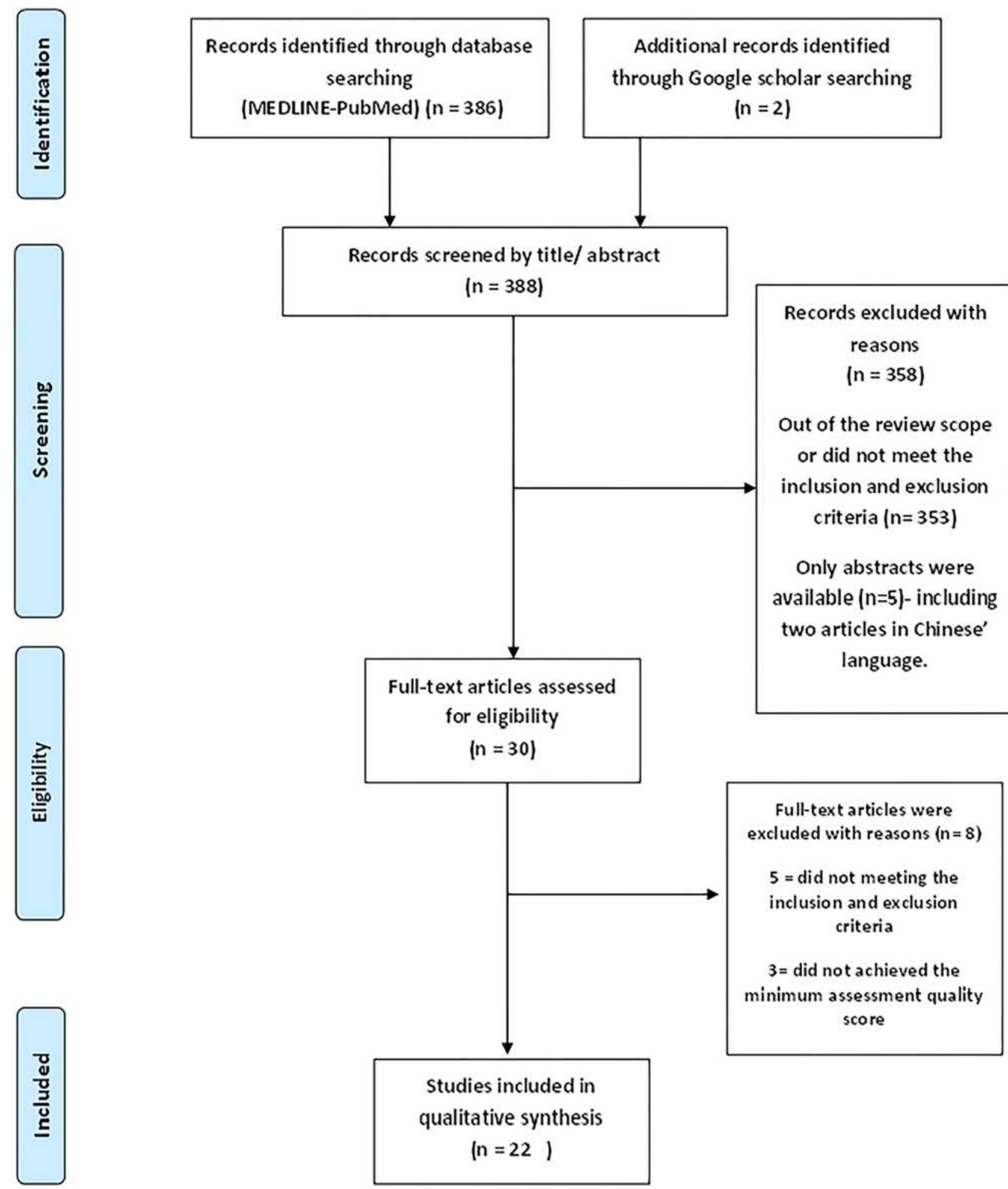

Studies included in qualitative synthesis

$$
\text { ( } n=22 \text { ) }
$$

Figure I Flow chart of the literature search and selection process.

\section{Quality Assessment}

Based on a six-point scale by Hayden et $a 1^{30}$ the following criteria was used to evaluate the quality of each selected study: (1) study participation (the sample is large enough
Additional records identified through Google scholar searching ( $\mathrm{n}=2$ )

Out of the review scope or did not meet the clusion and exclusion criteria $(n=353)$

Only abstracts were available ( $\mathrm{n}=5)$ - including language.

Full-text articles were

$\mathbf{5}=$ did not meeting the 
confounding factors control; (5) outcome measurement (full explanation of the method used for outcome measurement in such a way that reduces measurement bias; and (6) appropriateness of statistical analysis. One point score was given for each criterion; the score of $0-3$ points indicated low quality studies, while scores above 3-6 were considered high-quality studies.

\section{Results}

A primary search by MEDLINE/PubMed identified 388 articles; two extra articles were identified by Google Scholar search. By screening the titles and the abstracts, 358 articles were excluded for reasons of scope and/or not meeting the inclusion and exclusion criteria of the review. A total of 30 full-text articles were retrieved and analyzed for eligibility; of these, eight articles were excluded because five of them did not meet the inclusion and exclusion criteria and three articles did not achieve a minimum quality assessment score $(\leq 3)$. Finally, 22 articles were included for the qualitative analysis in the current review (Figure 1).

The selected studies included women from 14 countries: Poland, ${ }^{31}$ Czech Republic, ${ }^{28}$ Germany, ${ }^{16,32-34}$ Saudi Arabia, ${ }^{35,36}$ USA, ${ }^{20,37}$ Greece, ${ }^{24}$ Switzerland, ${ }^{23}$ Spain, ${ }^{38}$ Italy, ${ }^{18,27,39,40}$ Austria, ${ }^{41}$ UK, ${ }^{17}$ Japan,${ }^{42}$ Sweden, ${ }^{43}$ and Finland. ${ }^{44}$ These studies included cycling women with a wide age range (18-44 years), and the number of cases ranged from $\operatorname{six}^{17}$ to $259^{37}$ women. All studies were prospective observational studies, except four clinical studies that included multiple groups. ${ }^{16,39,43,44}$ In these four studies, leptin variation during menstrual cycle was determined on the control groups, which included spontaneous cycling women without any intervention; thus, they have been included in the present review. The majority of the studies had evaluated leptin during one complete menstrual cycle; however, two studies had evaluated leptin over three consecutive menstrual cycles ${ }^{16,32}$ and one study over two consecutive menstrual cycles. ${ }^{37}$ All studies measured leptin in the blood, except one study that evaluated leptin variation during menstrual cycle in a saliva sample. $^{33}$

\section{Variation of Leptin During Menstrual Cycle}

Of the included studies, 18 have shown significant changes in leptin level across different phases of the menstrual cycle $^{16-20,23,24,31-39,41,43}$ (Table 1), while four studies reported no changes in leptin throughout the menstrual cycle $^{27,28,42,44}$ (Table 2). Most of the studies reported significant changes in leptin and showed a steady increment from the lowest level at the early follicular phase to its peak at late luteal phase-except two studies, where leptin peak was detected at the mid-cycle phase near the time of LH surge. ${ }^{37,39}$

\section{Relationship Between Leptin Variation During Menstrual Cycle and the Hypothalamic-Pituitary-Gonadal Hormones}

The relationship of leptin during menstrual cycle with at least one of the hormones of the hypothalamic-pituitary-gonadal axis was evaluated by 17 studies, ${ }^{17-20,23,24,27,31,33-39,42,44}$ as seen in Tables 1-2.

\section{Effect of Obesity on Leptin Variation During Menstrual Cycle}

Among the selected studies, only four studies investigated the effect of obesity on leptin variation during menstrual cycle by comparing normal weight women with overweight/obese women. ${ }^{35,36,38,42}$ Two studies showed significant fluctuation of leptin level with the lowest values during the early follicular phase and the highest during the luteal phase in both normal weight and overweight/ obese groups. ${ }^{35,36}$ In contrast, one study detected leptin variation during the menstrual cycle in the normal group with higher levels during preovulatory and luteal phase, while overweight women showed stable leptin level. ${ }^{38}$ Another study reported no changes in leptin levels during the menstrual cycle in the normal weight group, while leptin showed significant variation during the luteal phase in the obese group. ${ }^{42}$

\section{Discussion}

The role of leptin in the reproductive function in humans is well documented. Leptin is considered to be the link between body fat storage and the hypothalamic-pituitary-gonadal axis. Many human and animal studies suggest that leptin has also been implicated in the physiology of puberty, menstrual cycle, menopause, pregnancy, and lactation. ${ }^{45}$ However, the role of leptin during the menstrual cycle showed some controversy. In addition, the mechanism describes the effect of obesity on leptin level during the menstrual cycle needs further review and analysis. 


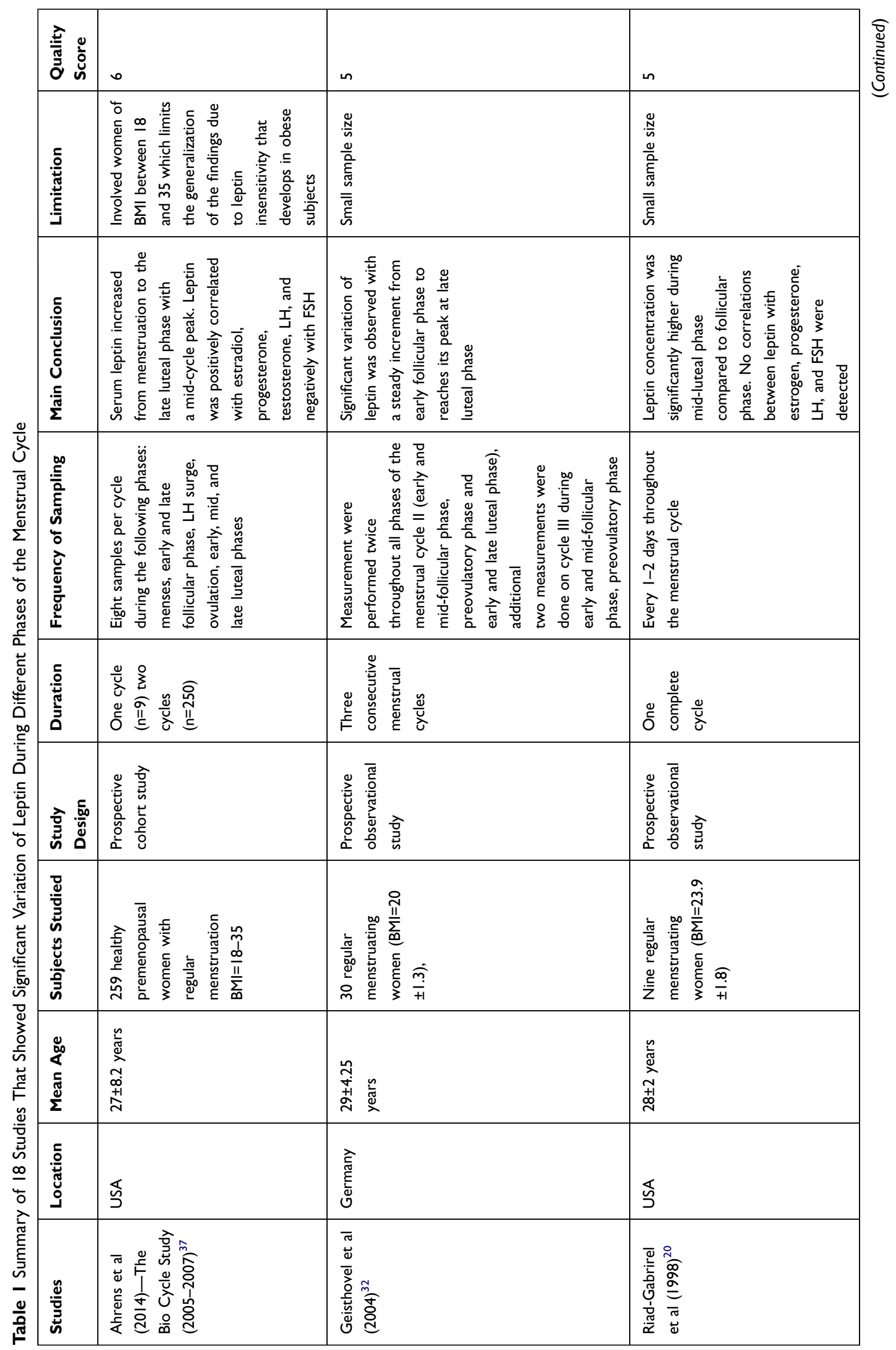




\begin{tabular}{|c|c|c|c|}
\hline 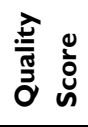 & in & $\stackrel{n}{+}$ & $\stackrel{n}{q}$ \\
\hline . & 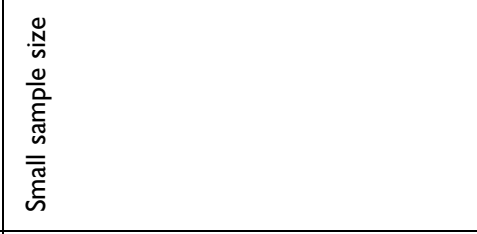 & 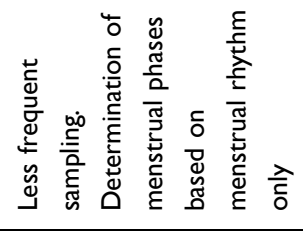 & 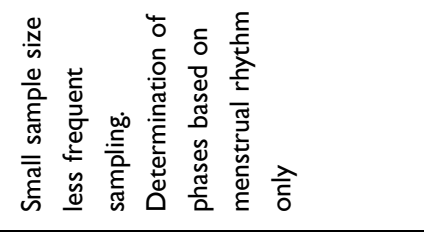 \\
\hline 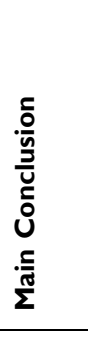 & 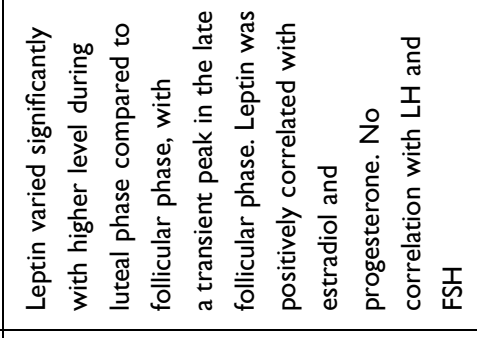 & 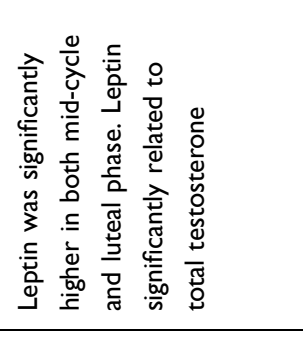 & 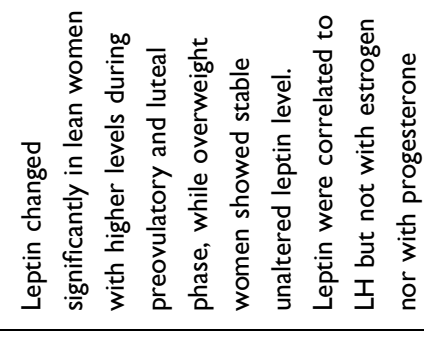 \\
\hline 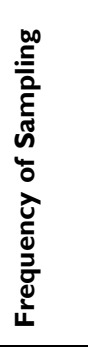 & 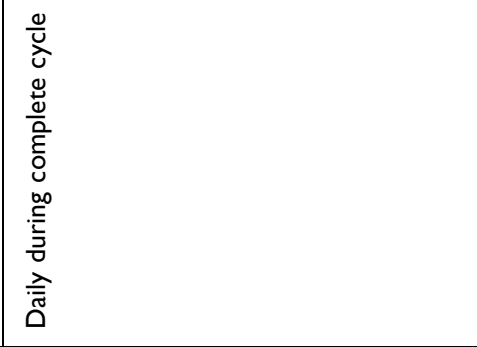 & 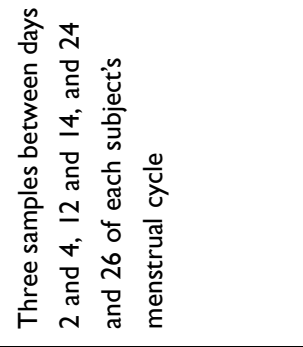 & 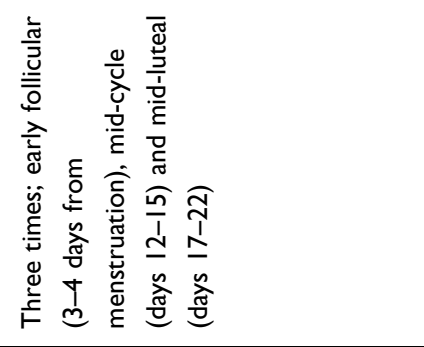 \\
\hline 产 & 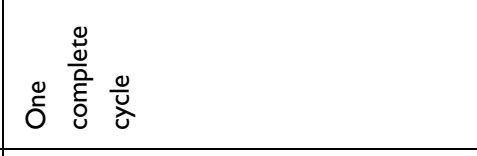 & 焉 & 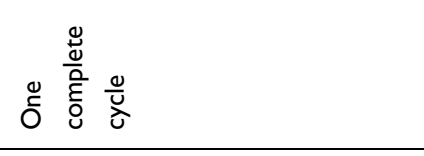 \\
\hline 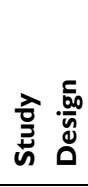 & 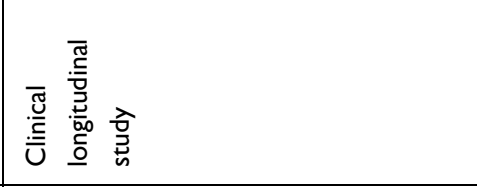 & 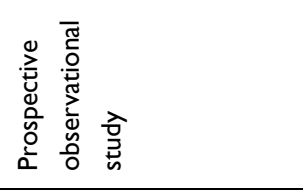 & 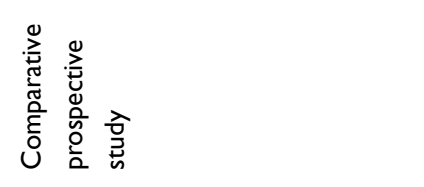 \\
\hline 总 & 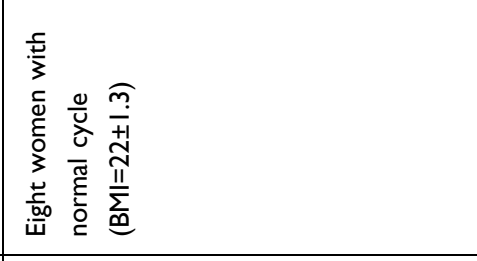 & 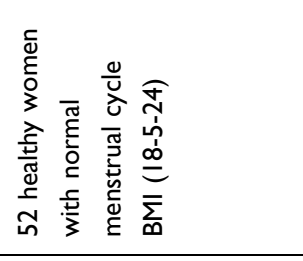 & 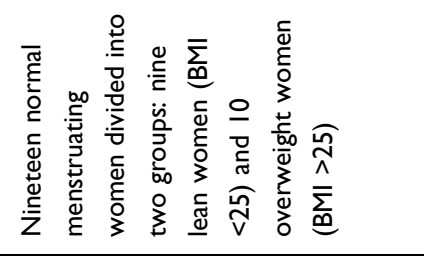 \\
\hline 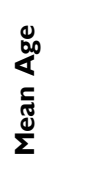 & 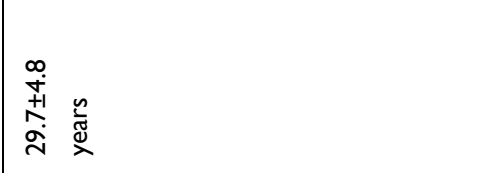 & 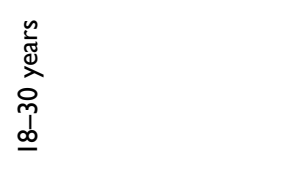 & 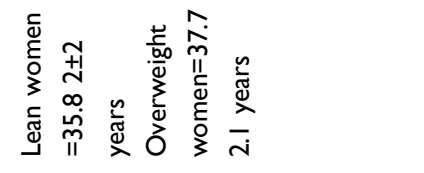 \\
\hline 递 & 츨 & $\begin{array}{l}\frac{D}{\tilde{E}} \\
\frac{0}{0}\end{array}$ & 䓌 \\
\hline 岕 & 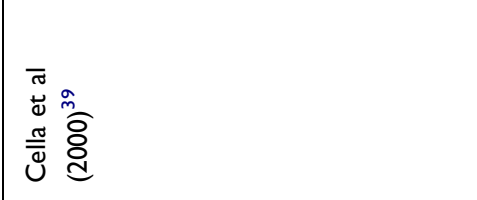 & 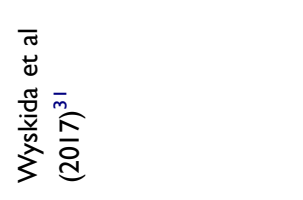 & 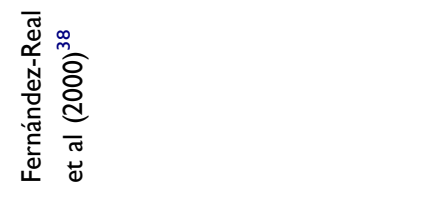 \\
\hline
\end{tabular}




\begin{tabular}{|c|c|c|c|}
\hline ซ & $\stackrel{n}{q}$ & $\stackrel{\leftrightarrow}{+}$ & $\stackrel{2}{+}$ \\
\hline 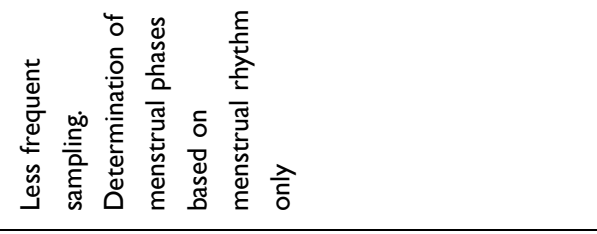 & 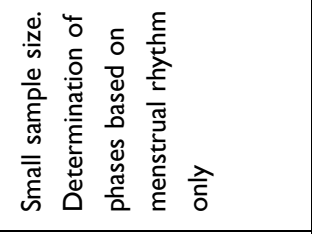 & 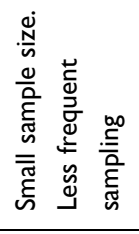 & 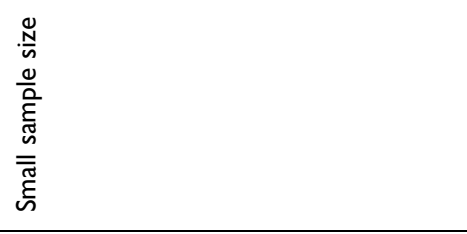 \\
\hline 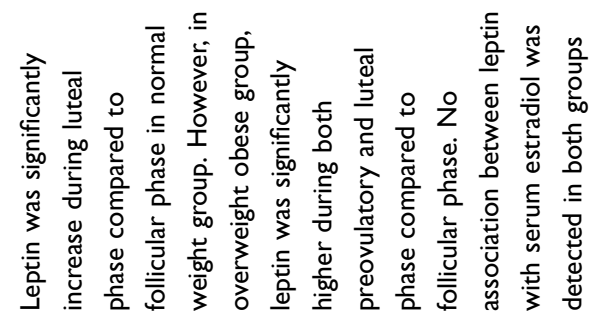 & 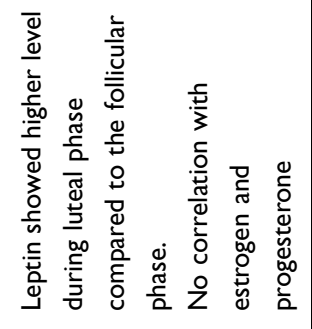 & 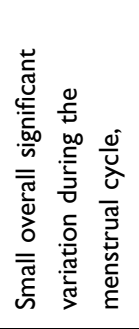 & 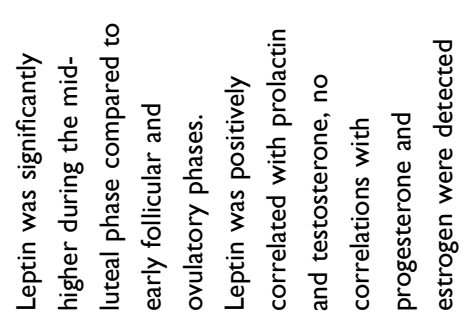 \\
\hline 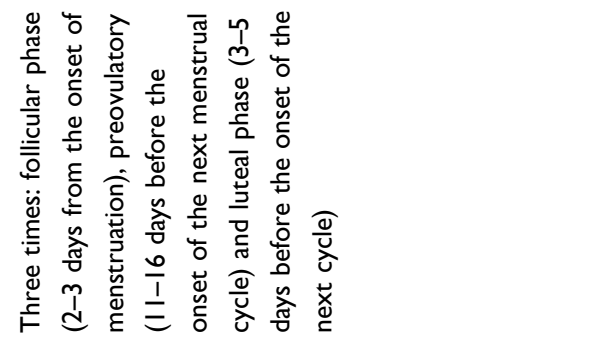 & 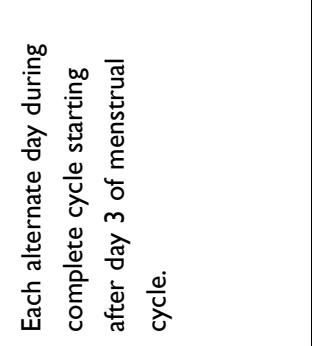 & 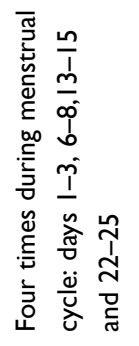 & 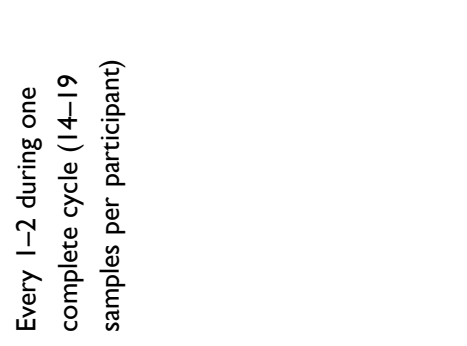 \\
\hline 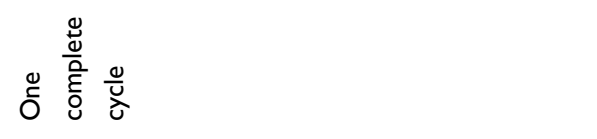 & 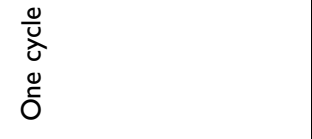 & 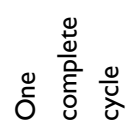 & 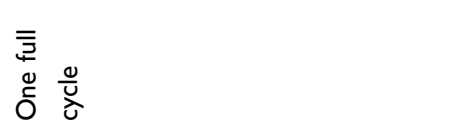 \\
\hline 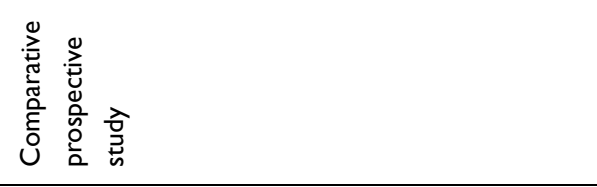 & 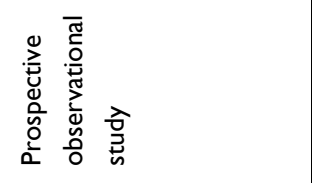 & 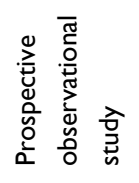 & 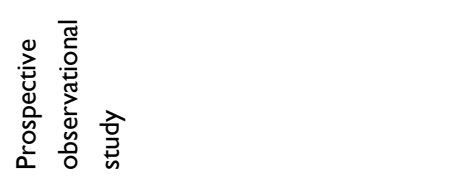 \\
\hline 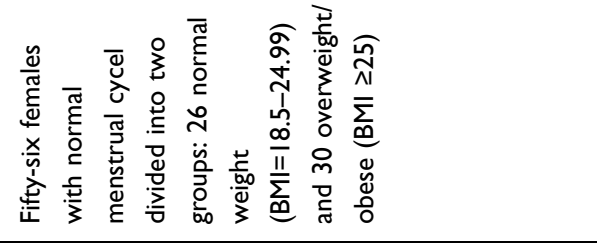 & 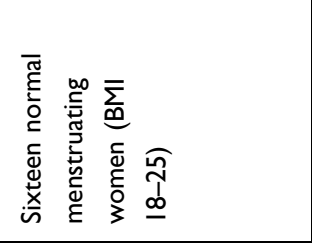 & 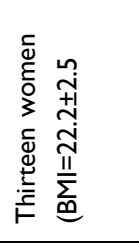 & 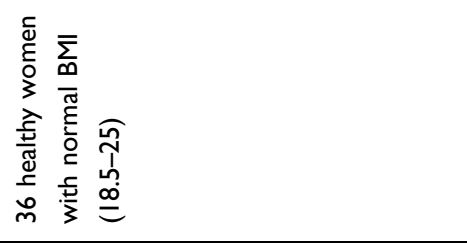 \\
\hline 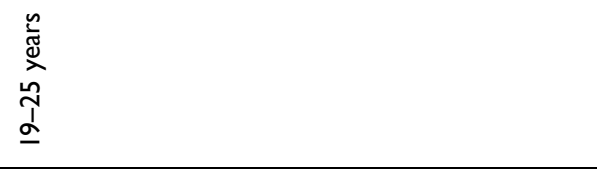 & 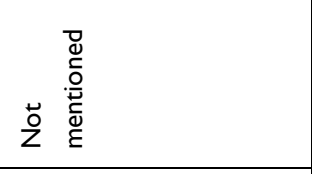 & 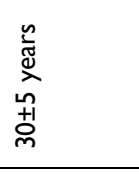 & 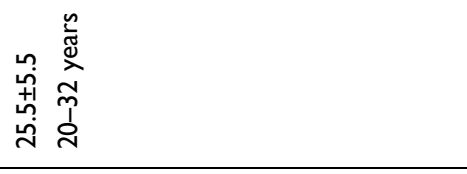 \\
\hline 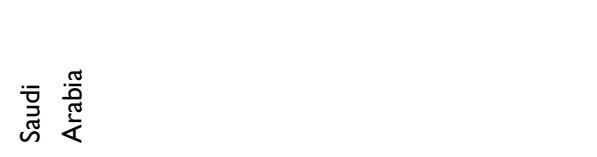 & 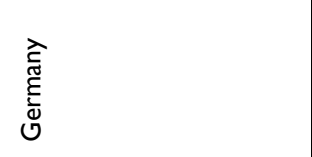 & 离 & 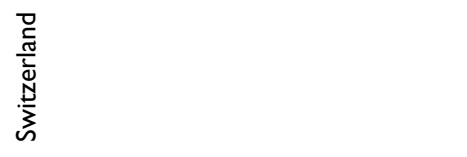 \\
\hline 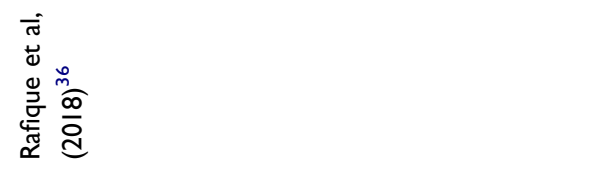 & 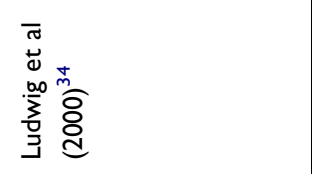 & 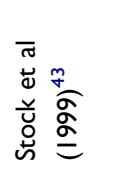 & 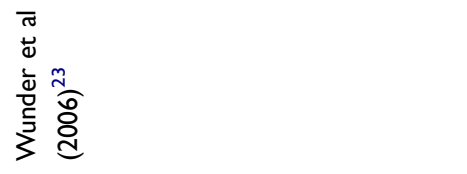 \\
\hline
\end{tabular}




\begin{tabular}{|c|c|c|c|}
\hline 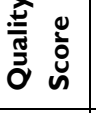 & $\stackrel{n}{+}$ & t & t \\
\hline & 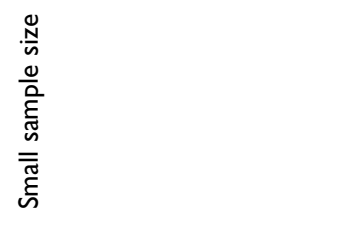 & 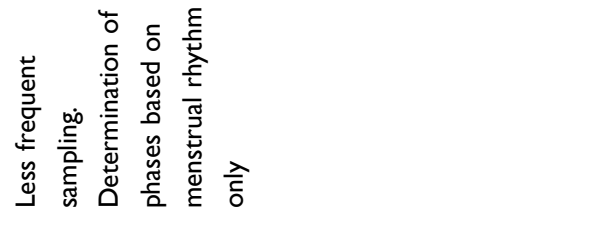 & 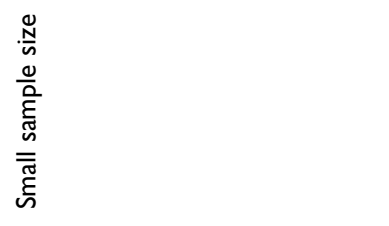 \\
\hline 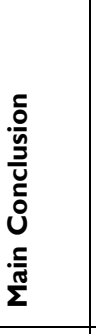 & 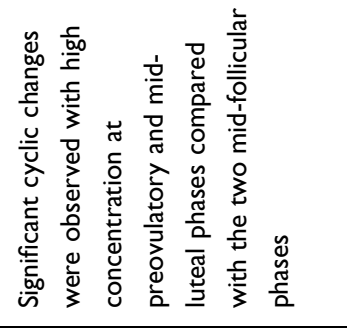 & 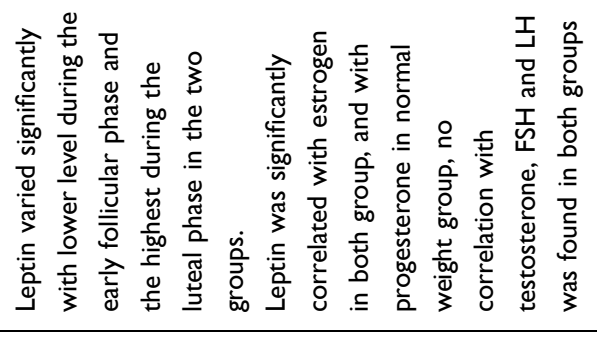 & 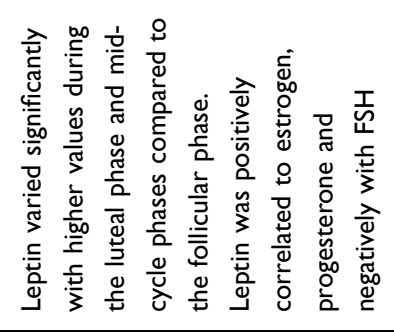 \\
\hline 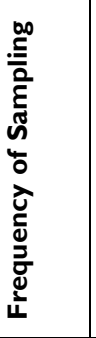 & 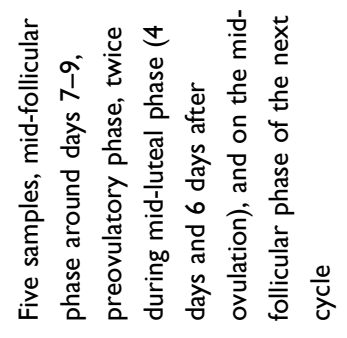 & 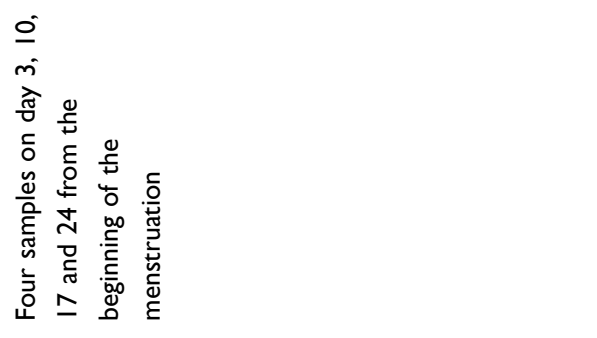 & 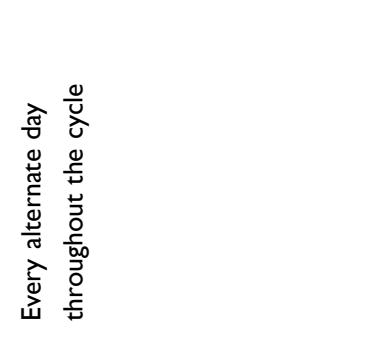 \\
\hline & 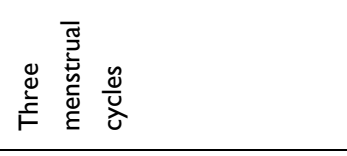 & 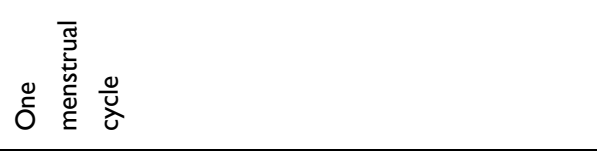 & 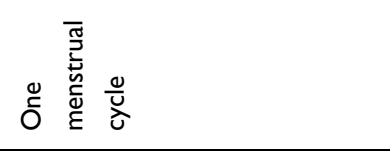 \\
\hline 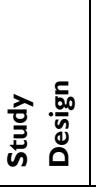 & 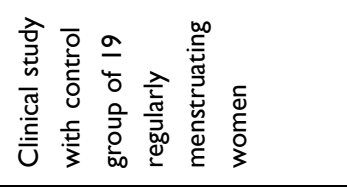 & 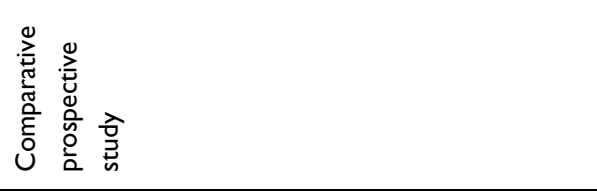 & 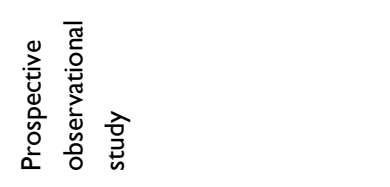 \\
\hline & 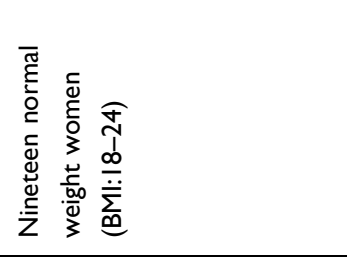 & 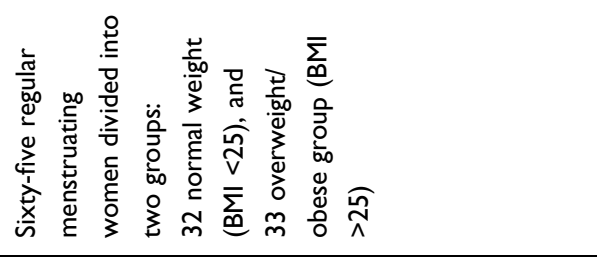 & 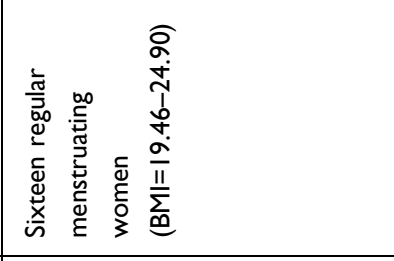 \\
\hline & 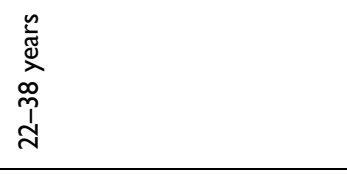 & 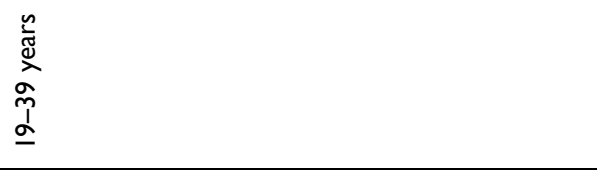 & 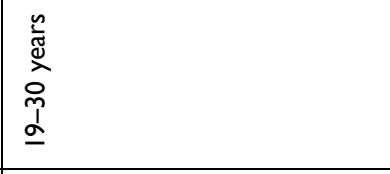 \\
\hline د.ّ & 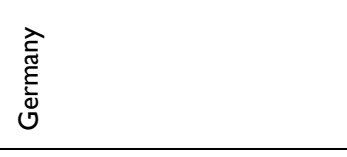 & 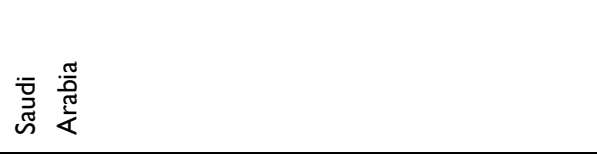 & 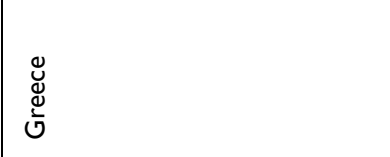 \\
\hline ڤั & 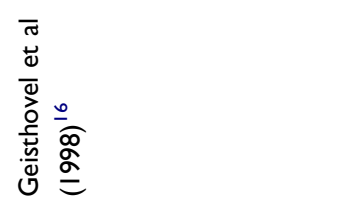 & 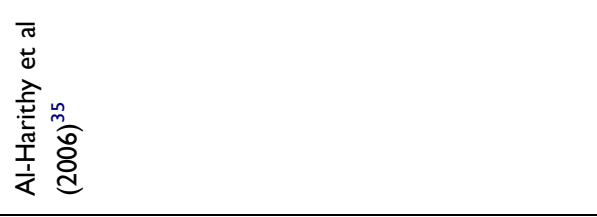 & 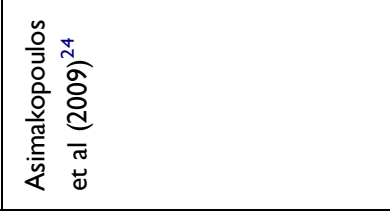 \\
\hline
\end{tabular}




\begin{tabular}{|c|c|c|c|}
\hline$\sigma$ & $\stackrel{\mathscr{n}}{m}$ & $\stackrel{n}{m}$ & $\stackrel{n}{m}$ \\
\hline 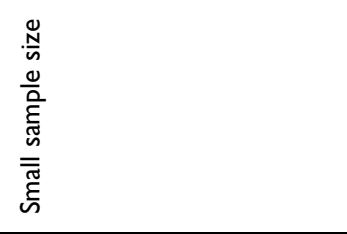 & 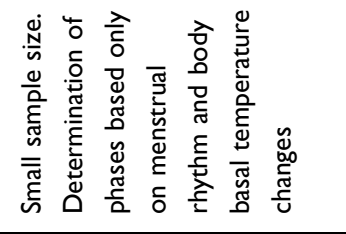 & 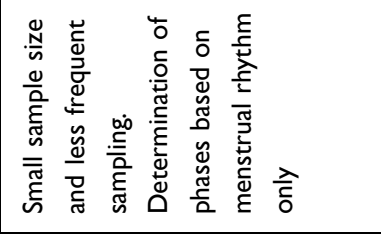 & 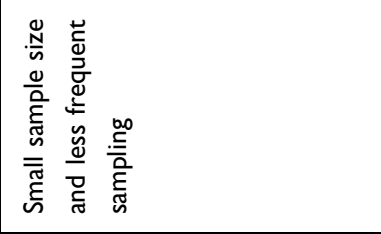 \\
\hline 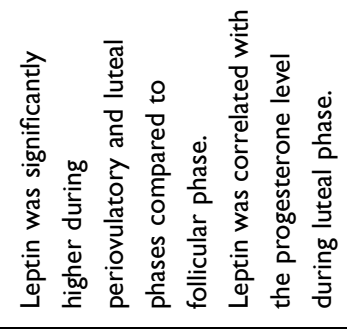 & 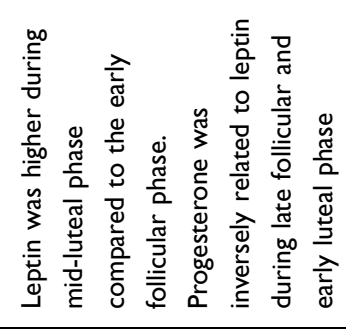 & 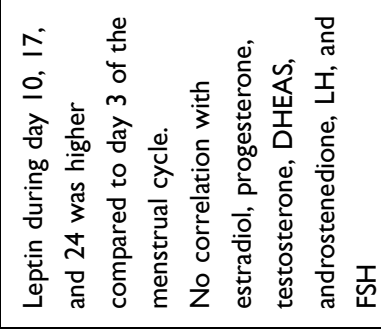 & 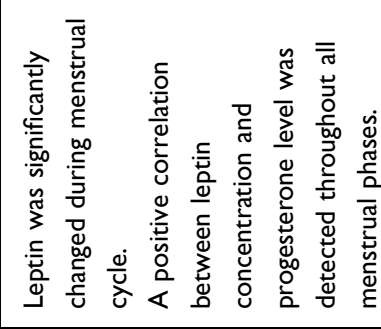 \\
\hline 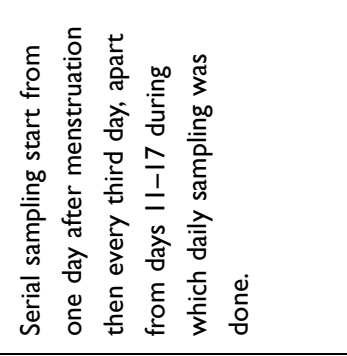 & 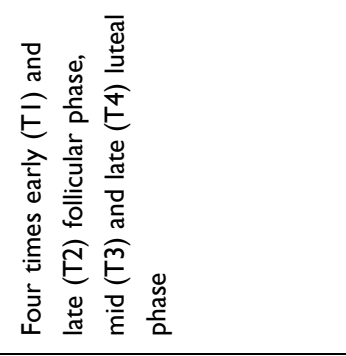 & 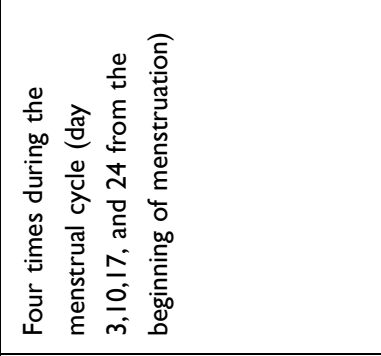 & 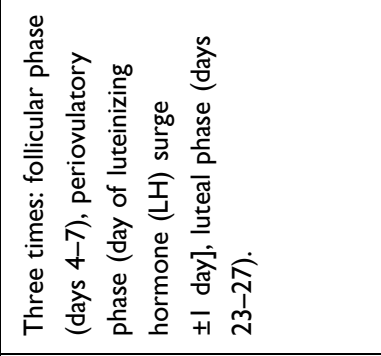 \\
\hline 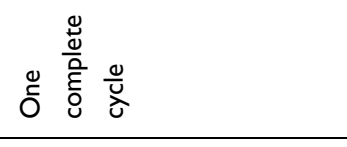 & 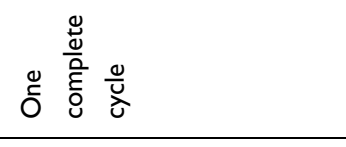 & 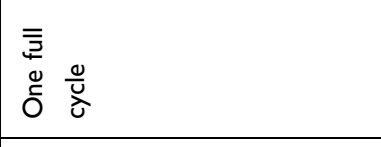 & 兽 \\
\hline 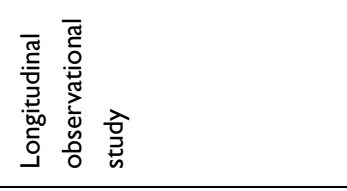 & 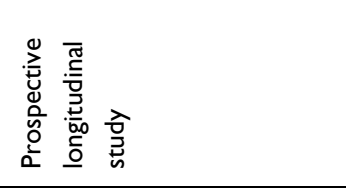 & 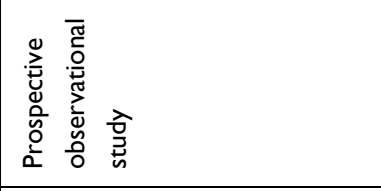 & 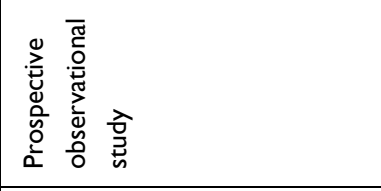 \\
\hline 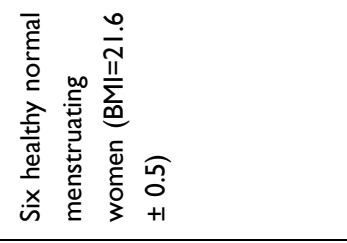 & 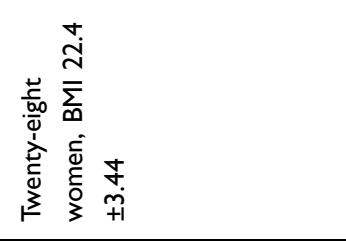 & 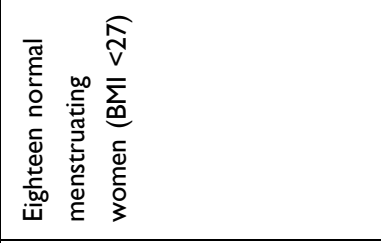 & 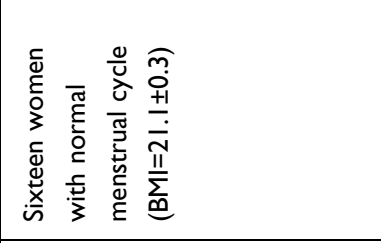 \\
\hline 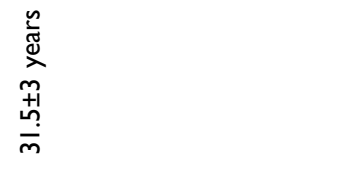 & 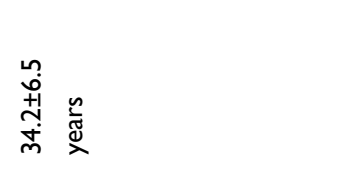 & 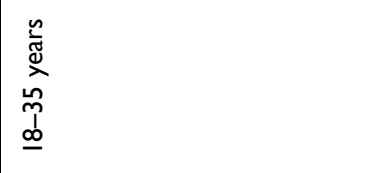 & 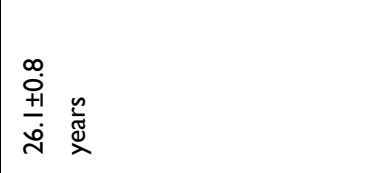 \\
\hline 弚 & 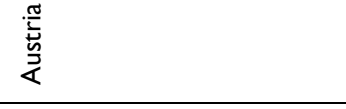 & 촢 & $\underline{\underline{\underline{\mathrm{I}}}}$ \\
\hline 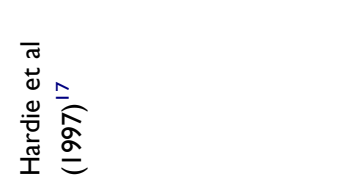 & 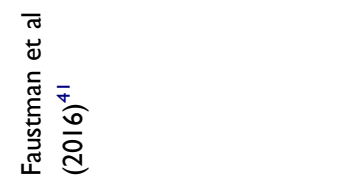 & 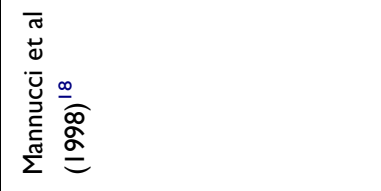 & 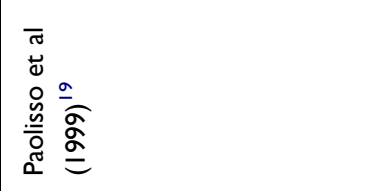 \\
\hline
\end{tabular}




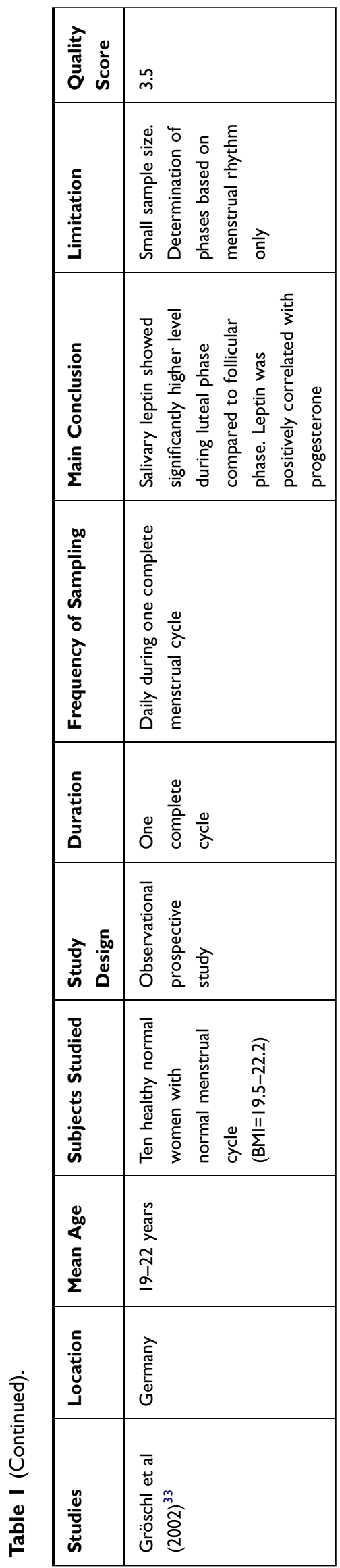

The majority of the studies evaluating leptin in normalweight, regularly menstruating females showed a significant variation during the menstrual cycle (Table 1). Only a few studies reported a small nonsignificant variation in leptin level across menstruation ${ }^{27,28,42,44}$ (Table 2). This insignificant variation could be attributed to the small sample size and method of determination of the menstrual phases in these studies, which is based on menstrual rhythm only. However, some of these studies reported a significant variation in leptin during the menstrual cycle in the obese group, ${ }^{42}$ while others showed high leptin levels in the ovulatory cycle group compared to the nonovulatory cycle ${ }^{27}$ and a positive correlation with LH level. ${ }^{44}$

The pattern of leptin variation during menstrual cycle is almost similar among all studies; leptin showed a high concentration during preovulatory and luteal phases when compared to the follicular phase with a steady increment starting from the follicular phase through the ovulatory phase and reaching a peak during the luteal phase (Table 1). However, two studies detected a peak of leptin just before ovulation, which coincides with LH surge (preovulatory peak), ${ }^{25,39}$ but this peak was not significantly different from the high luteal leptin level reported by these studies.

To control for the effect of adiposity on leptin variation during menstrual cycle, only normal weight women with BMI $<25$ were included in most of the studies. However, some studies had further demonstrated significant leptin variation during the menstrual cycle in overweight/obese women similar to the pattern observed in normal weight women. ${ }^{35,36,42}$ The presence of leptin variation in obese women indicates factors other than adiposity are implicated in leptin variation during menstruation. Corpus luteum formation during the luteinization phase could be possible cause of elevated leptin, and corpus luteum has been found to produce leptin in human ${ }^{46}$ and bovine models. ${ }^{47}$ Further support came from comparing ovulatory and anovulatory cycles; decreased leptin concentration was found during the anovulatory cycle (ie, without ovulation and corpus luteum) compared to the corresponding higher leptin level during the luteal phase of the ovulatory cycles (with ovulation and corpus luteum formation). ${ }^{25,27}$ In addition, the LH surge during ovulation stimulates the production of many inflammatory mediators within the ovarian follicles. ${ }^{48}$ Because leptin is considered a proinflammatory mediator, ${ }^{49}$ the high serum leptin during 


\begin{tabular}{|c|c|c|c|c|}
\hline & $m$ & $\stackrel{\wp}{+}$ & $\sigma$ & $\stackrel{n}{m}$ \\
\hline & 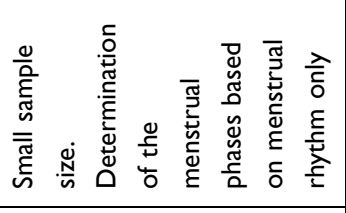 & 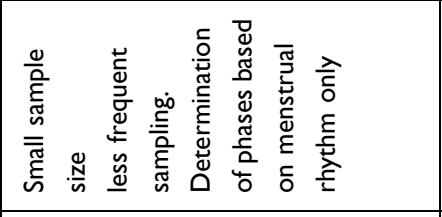 & 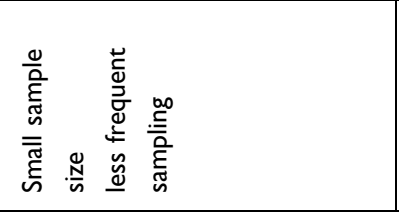 & 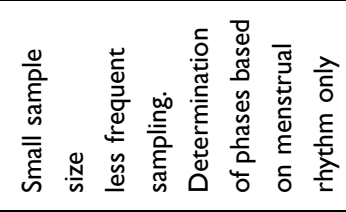 \\
\hline & 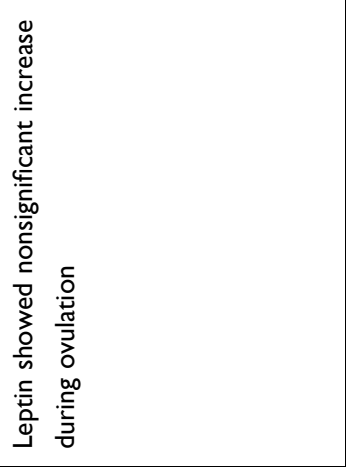 & 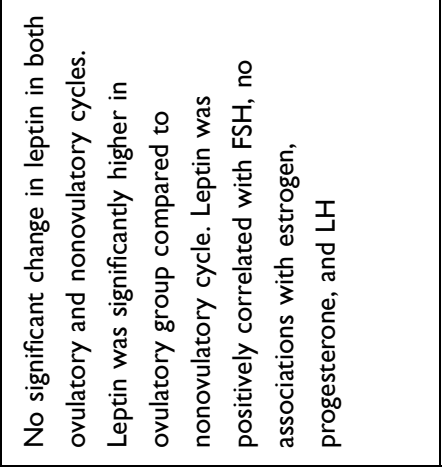 & 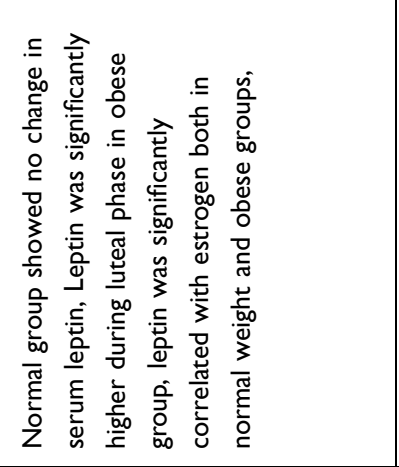 & 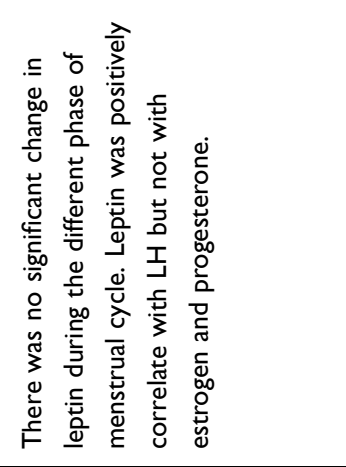 \\
\hline & 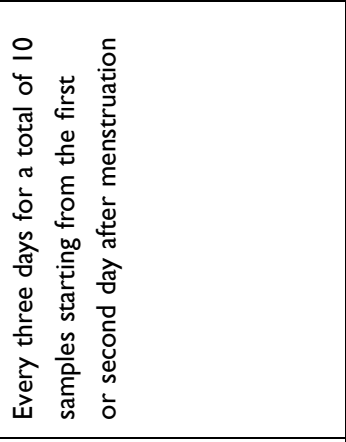 & 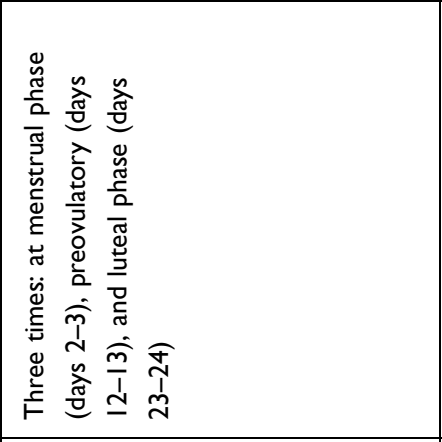 & 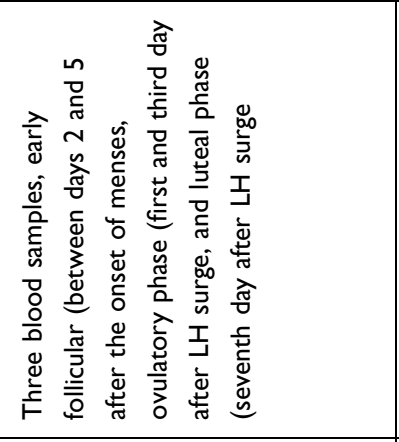 & 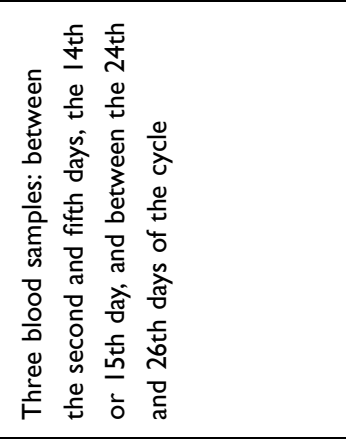 \\
\hline & 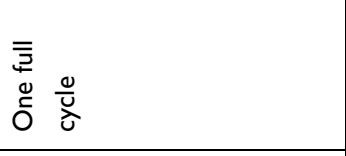 & 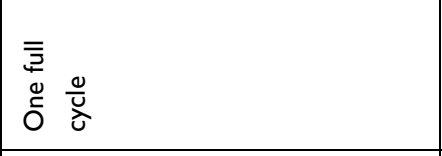 & 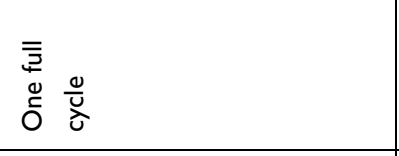 & 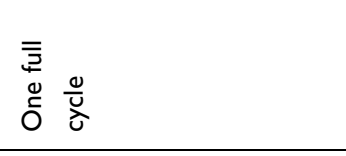 \\
\hline & 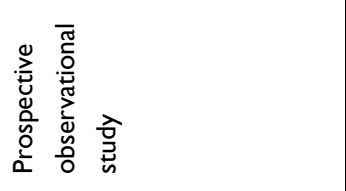 & 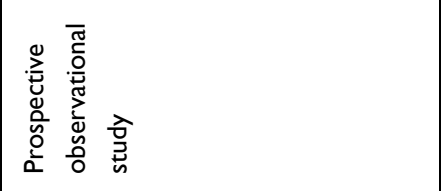 & 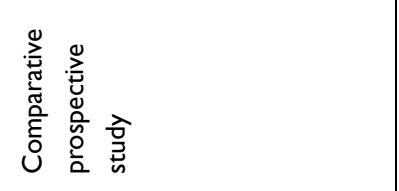 & 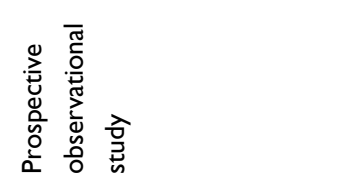 \\
\hline & 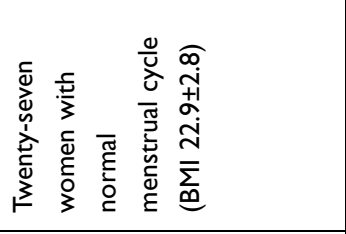 & 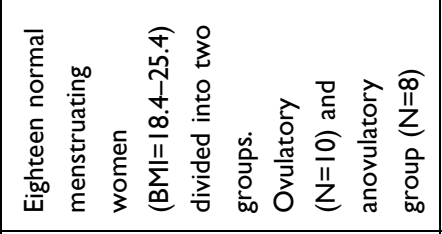 & 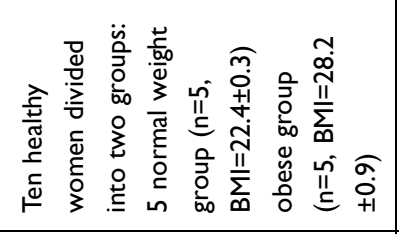 & 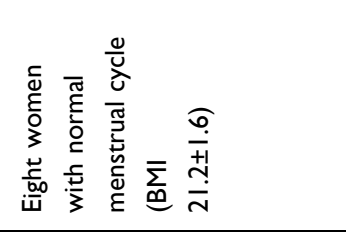 \\
\hline & 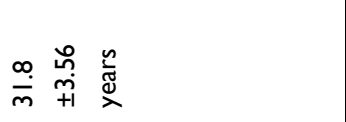 & 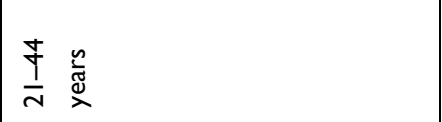 & 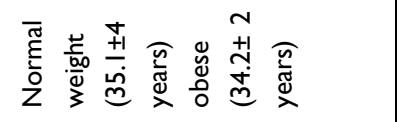 & 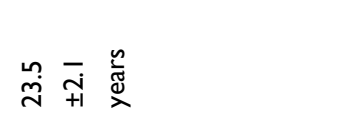 \\
\hline & 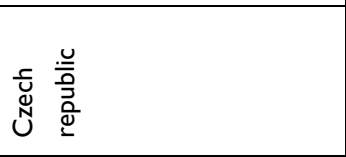 & 첲 & 䃈 & 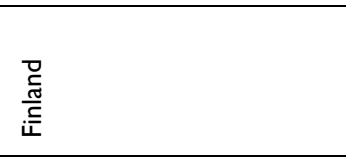 \\
\hline & 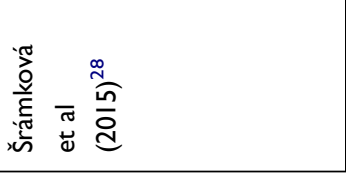 & 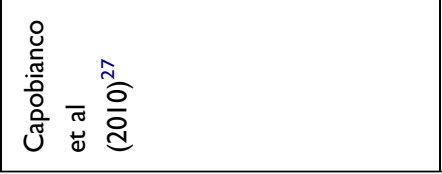 & 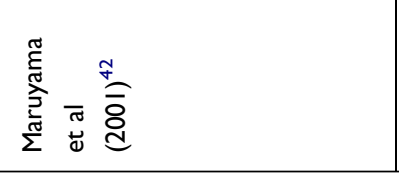 & 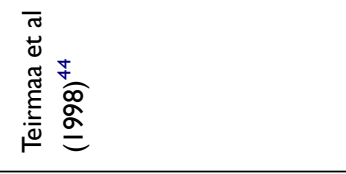 \\
\hline
\end{tabular}


preovulatory and the luteal phases might be related to inflammatory responses associated with ovulation.

Leptin produces a regulatory role on the menstrual cycle exerted at the hypothalamic-pituitary-gonadal axis both directly and indirectly. ${ }^{50}$ Since the GnRH neurons have no leptin receptors, the action of leptin on the hypothalamicpituitary-gonadal axis was found to be mediated through intermediate neurons. ${ }^{45}$ Intermediate neurons connected to the GnRH neurons on the hypothalamus and control the $\mathrm{GnRH}$ secretion through release of neuropeptides, such as proopiomelanocortin (POMC), neuropeptide Y (NPY), and kisspeptin. ${ }^{5,50,51}$ In addition, leptin could induce LH secretion directly from anterior pituitary. ${ }^{52,53}$ As further proof for the permissive action of leptin on the menstrual cycle, menstruation was restored by administration of recombinant leptin in females with hypothalamic amenorrhea. ${ }^{11,54}$

The literature on the relationship between leptin and female sex hormones during the menstrual cycle is inconsistent. Leptin was found to be positively correlated with estrogen and progesterone during the menstrual cycle in many studies. $^{17,19,24,33,35,37,39}$ In contrast, other studies reported no relationship of leptin levels with sex hormones during different menstrual phases. ${ }^{18,20,23,34,36,38}$ However, the absence of correlation between leptin and sex hormones reported by some studies does not mean an absence of leptin's role during the menstrual cycle. These controversial results might highlight a complex feedback loop controlling the interrelation between leptin and the hormones of hypothalamic-pituitary-gonadal axis, instead of a simple unidirectional relation.

\section{Conclusion}

This review clearly reports a significant variation of leptin hormone across the menstrual cycle, with a significant interplay with other hypothalamic-pituitary-gonadal hormones. Further studies on the molecular mechanism of leptin action exerted upon the hypothalamus, pituitary, and the ovary are recommended using both animal and human models.

\section{Disclosure}

The author reports no conflicts of interest in this work.

\section{References}

1. Friedman JM. Leptin at 14 y of age: an ongoing story. Am J Clin Nutr. 2009;89(3):3. doi:10.3945/ajen.2008.26788B

2. Mantzoros CS, Magkos F, Brinkoetter M, et al. Leptin in human physiology and pathophysiology. Am J Physiol Endocrinol Metab. 2011;301(4):E567-84. doi:10.1152/ajpendo.00315.2011
3. Park HK, Ahima RS. Physiology of leptin: energy homeostasis, neuroendocrine function and metabolism. Metabolism. 2015;64 (1):24-34. doi:10.1016/j.metabol.2014.08.004

4. Michalakis K, Mintziori G, Kaprara A, Tarlatzis BC, Goulis DG. The complex interaction between obesity, metabolic syndrome and reproductive axis: a narrative review. Metabolism. 2013;62(4):457-478. doi:10.1016/j.metabol.2012.08.012

5. Pérez-Pérez A, Sánchez-Jiménez F, Maymó J, Dueñas JL, Varone C, Sánchez-Margalet V. Role of leptin in female reproduction. Clin Chem Lab Med. 2015;53(1):15-28. doi:10.1515/cclm-2014-0387

6. Ingalls AM, Dickie MM, Snell GD. Obese, a new mutation in the house mouse. J Hered. 1950;41(12):317-318. doi:10.1093/oxfordjournals.jhered.a106073

7. Comninos AN, Jayasena CN, Dhillo WS. The relationship between gut and adipose hormones, and reproduction. Hum Reprod Update. 2013;20(2):153-174. doi:10.1093/humupd/dmt033

8. Vázquez MJ, Romero-Ruiz A, Tena-Sempere M. Roles of leptin in reproduction, pregnancy and polycystic ovary syndrome: consensus knowledge and recent developments. Metabolism. 2015;64(1):79-91. doi:10.1016/j.metabol.2014.10.013

9. Chou SH, Mantzoros C. 20 years of leptin: role of leptin in human reproductive disorders. $J$ Endocrinol. 2014;223(1):T49. doi:10.1530/ JOE-14-0245

10. Nieuwenhuis D, Pujol-Gualdo N, Arnoldussen IAC, Kiliaan AJ. Adipokines: a gear shift in puberty. Obes Rev. 2020;21(6):30. doi:10.1111/obr.13005

11. Chou SH, Chamberland JP, Liu X, et al. Leptin is an effective treatment for hypothalamic amenorrhea. Proc Natl Acad Sci U S A. 2011;108(16):6585-6590. doi:10.1073/pnas.1015674108

12. Al-Badri MR, Zantout MS, Azar ST. The role of adipokines in gestational diabetes mellitus. Ther Adv Endocrinol Metab. 2015;6 (3):103-108. doi:10.1177/2042018815577039

13. Fedullo AL, Schiattarella A, Morlando M, et al. Mediterranean diet for the prevention of gestational diabetes in the Covid-19 Era: implications of Il-6 In diabesity. Int $J$ Mol Sci. 2021;22(3):3. doi:10.3390/ ijms 22031213

14. Rizzo G, Garzon S, Fichera M, et al. Vitamin D and gestational diabetes mellitus: Is there a link? Antioxidants. 2019;8(11):11. doi:10.3390/antiox 8110511

15. Chehab FF. 20 years of leptin: leptin and reproduction: past milestones, present undertakings, and future endeavors. $J$ Endocrinol. 2014;223(1):T37-48. (). doi:10.1530/JOE-14-0413

16. Geisthövel F, Meysing A, Brabant G. C-peptide and insulin, but not C19-steroids, support the predictive value of body mass index on leptin in serum of premenopausal women. Hum Reprod. 1998;13 (3):547-553. doi:10.1093/humrep/13.3.547

17. Hardie L, Trayhurn P, Abramovich D, Fowler P. Circulating leptin in women: a longitudinal study in the menstrual cycle and during pregnancy. Clin Endocrinol. 1997;47(1):101-106. doi:10.1046/ j.1365-2265.1997.2441017.x

18. Mannucci E, Ognibene A, Becorpi A, et al. Relationship between leptin and oestrogens in healthy women. Eur J Endocrinol. 1998;139 (2):198-201. doi:10.1530/eje.0.1390198

19. Paolisso G, Rizzo MR, Mazziotti G, et al. Lack of association between changes in plasma leptin concentration and in food intake during the menstrual cycle. Eur J Clin Invest. 1999;29(6):490-495. doi:10.1046/j.1365-2362.1999.00488.x

20. Riad-Gabriel MG, Jinagouda SD, Sharma A, Boyadjian R, Saad MF. Changes in plasma leptin during the menstrual cycle. Eur J Endocrinol. 1998;139(5):528-531. doi:10.1530/ eje. 0.1390528

21. Ajala OM, Ogunro PS, Elusanmi GF, Ogunyemi OE, Bolarinde AA. Changes in serum leptin during phases of menstrual cycle of fertile women: relationship to age groups and fertility. Int $J$ Endocrinol Metab. 2013;11(1):27-33. doi: $10.5812 /$ ijem. 6872 
22. Einollahi N, Dashti N, Nabatchian F. Serum leptin concentrations during the menstrual cycle in Iranian healthy women. Acta Med Iran. 2010;48(5):300-303.

23. Wunder DM, Yared M, Bersinger NA, Widmer D, Kretschmer R, Birkhäuser MH. Serum leptin and C-reactive protein levels in the physiological spontaneous menstrual cycle in reproductive age women. Eur J Endocrinol. 2006;155(1):137-142. doi:10.1530/eje.1.02178

24. Asimakopoulos B, Milousis A, Gioka T, et al. Serum pattern of circulating adipokines throughout the physiological menstrual cycle. Endocr J. 2009;56(3):425-433. doi:10.1507/endocrj.K08E-222

25. Ahrens K, Mumford SL, Schliep KC, et al. Serum leptin levels and reproductive function during the menstrual cycle. Am J Obstet Gynecol. 2014;210(3):248e1-9. doi:10.1016/j.ajog.2013.11.009

26. Lin KC. Changes of circulating leptin levels during normal menstrual cycle: relationship to estradiol and progesterone. Kaohsiung $\mathrm{J} \mathrm{Med}$ Sci. 1999;15(10):597-602.

27. Capobianco G, de Muro P, Cherchi GM, et al. Plasma levels of C-reactive protein, leptin and glycosaminoglycans during spontaneous menstrual cycle: differences between ovulatory and anovulatory cycles. Arch Gynecol Obstet. 2010;282(2):207-213. doi:10.1007/s00404-010-1432-2

28. Šrámková M, Dušková $M$, Vítků J, et al. Levels of adipokines and some steroids during the menstrual cycle. Physiol Res. 2015;64(Suppl 2):933116

29. Page MJ, Moher D, Bossuyt PM, Boutron I, Hoffmann TC, Mulrow CD, et al. PRISMA 2020 explanation and elaboration: updated guidance and exemplars for reporting systematic reviews. BMJ. 2021;372n160. doi:10.1136/bmj.n160

30. Hayden JA, Côté P, Bombardier C. Evaluation of the quality of prognosis studies in systematic reviews. Ann Intern Med. 2006;144 (6):427-437. doi:10.7326/0003-4819-144-6-200603210-00010

31. Wyskida K, Franik G, Wikarek T, et al. The levels of adipokines in relation to hormonal changes during the menstrual cycle in young, normal-weight women. Endocr Connect. 2017;6(8):892-900. doi:10.1530/EC-17-0186

32. Geisthövel F, Jochmann N, Widjaja A, Horn R, Brabant G. Serum pattern of circulating free leptin, bound leptin, and soluble leptin receptor in the physiological menstrual cycle. Fertil Steril. 2004;81 (2):398-402. doi:10.1016/j.fertnstert.2003.06.020

33. Gröschl M, Rauh M, Dörr HG, Blum WF, Rascher W, Dötsch J. Salivary leptin levels during the menstrual cycle and their relation to progesterone. Fertil Steril. 2002;77(6):1306-1307. doi:10.1016/ s0015-0282(02)03095-9

34. Ludwig M, Klein HH, Diedrich K, Ortmann O. Serum leptin concentrations throughout the menstrual cycle. Arch Gynecol Obstet. 2000;263(3):99-101. doi:10.1007/s004040050004

35. Al-Harithy RN, Al-Doghaither H, Abualnaja K. Correlation of leptin and sex hormones with endocrine changes in healthy Saudi women of different body weights. Ann Saudi Med. 2006;26(2):110-115. doi:10.5144/0256-4947.2006.110

36. Rafique N, Salem AM, Latif R, Al MH. Serum leptin level across different phases of menstrual cycle in normal weight and overweight/ obese females. Gynecol Endocrinol. 2018;34(7):601-604. doi:10.1080/09513590.2017.1419173

37. Ahrens K, Mumford SL, Schliep KC, et al. Serum leptin levels and reproductive function during the menstrual cycle. Am J Obstet Gynecol. 2014;210(3):8. doi:10.1016/j.ajog.2013.10.043

38. Fernández-Real JM, Gutierrez C, Vendrell J, Casamitjana R, Ricart W. Plasma soluble tumor necrosis factor-alpha receptors circulate in proportion to leptin levels during the menstrual cycle in lean but not in obese women. Eur J Endocrinol. 2000;143(2):235-241. doi:10.1530/eje. 0.1430235
39. Cella F, Giordano G, Cordera R. Serum leptin concentrations during the menstrual cycle in normal-weight women: effects of an oral triphasic estrogen-progestin medication. Eur $J$ Endocrinol. 2000;142(2):174-178. doi:10.1530/eje.0.1420174

40. Messinis IE, Milingos S, Zikopoulos K, Kollios G, Seferiadis K, Lolis D. Leptin concentrations in the follicular phase of spontaneous cycles and cycles superovulated with follicle stimulating hormone. Hum Reprod. 1998;13(5):1152-1156. doi:10.1093/humrep/13.5.1152

41. Faustmann G, Tiran B, Maimari T, et al. Circulating leptin and NF$\kappa \mathrm{B}$ activation in peripheral blood mononuclear cells across the menstrual cycle. Biofactors. 2016;42(4):376-387. doi:10.1002/biof.1281

42. Maruyama S, Minami S, Kaseki H, Ishihara K, Araki S, Suzue R. A comparison of serum leptin concentrations in obese and normal weight Japanese women with regular menstrual cycle. J Nutr Sci Vitaminol. 2001;47(1):87-89. doi:10.3177/jnsv.47.87

43. Stock SM, Sande EM, Bremme KA. Leptin levels vary significantly during the menstrual cycle, pregnancy, and in vitro fertilization treatment: possible relation to estradiol. Fertil Steril. 1999;72 (4):657-662. doi:10.1016/S0015-0282(99)00321-0

44. Teirmaa T, Luukkaa V, Rouru J, Koulu M, Huupponen R. Correlation between circulating leptin and luteinizing hormone during the menstrual cycle in normal-weight women. Eur J Endocrinol. 1998;139 (2):190-194. doi:10.1530/eje.0.1390190

45. Chehab FF. 20 years of leptin: leptin and reproduction: past milestones, present undertakings, and future endeavors. J Endocrinol. 2014;223(1):14-0413.

46. Zendron C, Goncalves HF, Cavalcante FS, et al. Increased expression of the leptin receptor in human ovaries affected by endometrioma and detection of high levels of leptin in the ovarian endometriomal fluid. J Ovarian Res. 2014;7(1):2. doi:10.1186/1757-2215-7-2

47. Sarkar M, Schilffarth S, Schams D, Meyer HH, Berisha B. The expression of leptin and its receptor during different physiological stages in the bovine ovary. Mol Reprod Dev. 2010;77(2):174-181. doi: $10.1002 / \mathrm{mrd} .21129$

48. Richards JS, Pangas SA. New insights into ovarian function. Handb Exp Pharmacol. 2010;(198):3-27. Epub 2010/ 09/15.

49. Scotece M, Conde J, Lopez V, et al. Adiponectin and leptin: new targets in inflammation. Basic Clin Pharmacol Toxicol. 2014;114 (1):97-102. doi:10.1111/bcpt.12109

50. Garcia-Galiano D, Allen SJ, Elias CF. Role of the adipocyte-derived hormone leptin in reproductive control. Horm Mol Biol Clin Investig. 2014;19(3):141-149. doi:10.1515/hmbci-2014-0017

51. Tena-Sempere M. Interaction between energy homeostasis and reproduction: central effects of leptin and ghrelin on the reproductive axis. Horm Metab Res. 2013;45(13):919-927. doi:10.1055/s-00331355399

52. Dagklis T, Kouvelas D, Kallaras K, et al. Leptin increases luteinizing hormone secretion of fasting female rats. Clin Exp Obstet Gynecol. 2015;42(1):18-21.

53. Kirsz K, Szczesna M, Dudek K, Bartlewski PM, Zieba DA. Influence of season and nutritional status on the direct effects of leptin, orexin-A and ghrelin on luteinizing hormone and growth hormone secretion in the ovine pituitary explant model. Domest Anim Endocrinol. 2014;48:69-76. doi:10.1016/j.domaniend.2014.02.005

54. Welt CK. Will leptin become the treatment of choice for functional hypothalamic amenorrhea? Nat Clin Pract Endocrinol Metab. 2007;3 (8):556-557. doi:10.1038/ncpendmet0561 


\section{Publish your work in this journal}

The International Journal of Women's Health is an international, peerreviewed open-access journal publishing original research, reports, editorials, reviews and commentaries on all aspects of women's healthcare including gynecology, obstetrics, and breast cancer. The manuscript management system is completely online and includes a very quick and fair peer-review system, which is all easy to use. Visit http://www.dovepress.com/testimonials.php to read real quotes from published authors.

Submit your manuscript here: https://www.dovepress.com/international-journal-of-womens-health-journal 